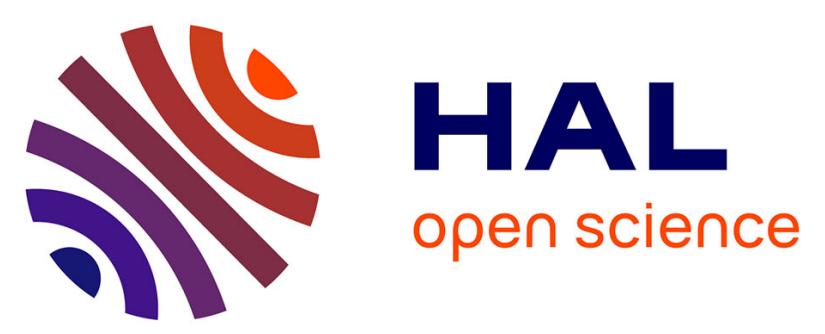

\title{
Disparités spatiales et financement des biens et services publics de proximité en Haïti. Le cas des budgets communaux de 2017-2018
}

Christophe Providence

\section{- To cite this version:}

Christophe Providence. Disparités spatiales et financement des biens et services publics de proximité en Haïti. Le cas des budgets communaux de 2017-2018. 30th annual conference of Haitian Studies Association: Haitian Studies at the Crossroads: Integrating the Humanities, Arts, Religions, Technology, and Sciences, Nov 2018, Port-au-Prince, Haïti. hal-02124721

\section{HAL Id: hal-02124721 https://hal.univ-antilles.fr/hal-02124721}

Submitted on 9 May 2019

HAL is a multi-disciplinary open access archive for the deposit and dissemination of scientific research documents, whether they are published or not. The documents may come from teaching and research institutions in France or abroad, or from public or private research centers.
L'archive ouverte pluridisciplinaire HAL, est destinée au dépôt et à la diffusion de documents scientifiques de niveau recherche, publiés ou non, émanant des établissements d'enseignement et de recherche français ou étrangers, des laboratoires publics ou privés. 


\title{
"Disparités spatiales et financement des biens et services publics de proximité en Haïti. Le cas des budgets communaux de 2017-2018"
}

Dr Christophe PROVIDENCE

Membre du CREGED

\begin{abstract}
Résumé
Pendant longtemps en Haïti, le développement fut pensé comme l'apanage des autorités centrales qui ont conçu, dans des logiques nationales, des stratégies à cet effet sans forcément prendre en compte les spécificités infranationales. Cette vision de mise en projet des territoires a émergé d'un contexte et d'une histoire uniques qui impliqueraient dès lors une logique de développement unique. En prenant le cas d'études les allocations budgétaires aux communes haïtiennes pour l'année fiscal 2017-2018, nous voulons démontrer que la logique de répartition du financement des collectivités territoriales n'est ni neutre ni stratégique. L'objectif de cet article est double. D'une part, c'est de modéliser les disparités spatiales et l'instabilité structurelle sur le territoire national. D'autre part, c'est d'étudier les variations dans l'espace et d'expliquer le caractère inclusif et complémentaire du processus de développement territorial.

L'analyse spatiale des dotations communales de crédits budgétaires révèle deux grandes anomalies dans la logique de financement de ces territoires. La première anomalie considère la légitimation des disparités territoriales et socioéconomiques qui vient d'un choix non éclairé des dirigeants à concevoir le territoire haïtien et les relations de proximité entre les acteurs localisés. La seconde anomalie se retrouve dans le manque de vision ou de considération globale dans la stratégie nationale pour le développement territorial. L'Etat haïtien ne peut donc pas faire une gestion prévisionnelle et stratégique du territoire car il est trop dépendant des événements conjoncturels.
\end{abstract}

Mots clés: Développement territorial, disparités spatiales, proximités, financement local, imbrication territoriale 


\section{I- Partie introductive}

Le débat sur le développement local place les acteurs locaux et les spécificités territoriales au cœur du processus de transformation des territoires. Ce processus, ayant pour but ultime l'amélioration des conditions et de la qualité de vie de la population, implique la mobilisation des ressources pour la fourniture des biens et services publics de proximité (Pecqueur et Vicente, 2000 ;Providence, 2015). Deux dynamiques non-exclusives peuvent contribuer à la mobilisation de ces ressources. Il s'agit d'abord de la densification institutionnelle qui suppose la manière dont la production locale est réalisée pour créer de la richesse et financer des projets communs. Ensuite, on retrouve les actions volontaristes de transformation d'un territoire par les acteurs extérieurs à ce territoire (mobilisation de ressources techniques, financières, etc.). Cette approche renvoie vers d'autres horizons comme celui de la diplomatie internationale où les peuples semblent interagir les uns sur les autres dans la planification du développement territorial.

Pendant longtemps en Haïti, le développement fut pensé comme l'apanage des autorités centrales qui ont conçu, dans des logiques nationales, des stratégies à cet effet sans forcément prendre en compte les spécificités infranationales. Plusieurs raisons expliquent aujourd'hui,malgré l'adoption de la décentralisation comme moyen de redynamiser les échelons nationaux, que les disparités spatiales témoignent de l'absencede volonté politiquedans les choix collectifs de développement. Si l'on admet (dans les discours) le principe de participation locale dans la mise en projet des territoires, les expériences des trente dernières années prouvent qu'il s'agit d'un vœu creux.

Cette vision de mise en projet des territoiresa émergé d'un contexte et d'une histoire uniques (avec la Constitution de 1987) qui impliqueraient dès lors une logique de développement unique (Providence, 2015). Ainsi, le développement local est avant tout l'affaire d'acteurs de toutes sortes, mis en rapport, mobilisés en vue de stimuler une synergie créatrice et porteuse d'effet de développement (Le Loup et al., 2003).

Ce papier cherche à analyser le processus de financement des collectivités communales haïtiennes responsablesde la planification du développement territorial. Or cette responsabilité impose la fourniture de biens et services publics de proximité aux populations locales qui contribuent par les mécanismes des Lois de finance locale au financement de leurs territoires. Dans les recherches sur le sous-développement d'Haïticet instrument financier n'a jamais été analysé comme outil stratégique de lutte contre les disparités socioéconomiques et territoriales (Providence, 2015). Se pose alors deux principes fondamentaux : le principe du financement des collectivités territoriales et celui de leur imbrication qui suppose la mise en concurrence des territoires. Ces principes invitent à considérer le niveau d'agrégation intermédiaire des départements et des communes qui sont pris dans des relations des voisinages tant par rapport au caractère transversal de certains problèmes communs que par les spécificités de chaque territoire communal.

En fait, il s'agit pour nous d'aborder la problématique du financement des Collectivités Territoriales haïtiennes se basant deux phénomènes simultanés :

1. L'autocorrélation dans le choix de financement des territoires, dans le sens d'une imbrication territoriale, qui atteste d'une stratégie de l'Etat pour le financement du développement national ; 
2. L'hétérogénéité dans les choix d'allocation budgétaire pour le financement de chaque territoire communal ou départemental, c'est-à-dire le financement spécifiquedes projets spatialement distribués.

En prenant le cas d'études les allocations des crédits budgétaires aux communes haïtiennes pour l'année fiscal 2017-2018, nous voulons démontrer que la logique de répartition du financement des collectivités territoriales n'est ni neutre ni stratégique. Pour cela, il s'agit de mobiliser des outils de l'économétrie spatiale afin de modéliser les deux phénomènes susmentionnés, comme c'est le cas dans de nombreux ouvrages : Cliff et Ord (1981), Upton et Fingleton (1985), Griffith (1988), Anselin (1988a), Anselin (1988b), Anselin et Griffith (1988), Haining (1990), Cressie (1993), Bailey et Gatrell (1995), Anselin et Bera (1998), Le Gallo (2000), Le Gallo et Ertur (2000), Fotheringham, Brundson et al. (2000).

L'objectif de cet article est double. D'une part, le fait de travailler sur des données géographiques concernant les budgets communaux et le financement des territoires par la Loi de finance de 2017-2018 nous permet de modéliser les disparités spatiales et l'instabilité structurelle sur le territoire national. Les statistiques spatiales traitées à partir du Logiciel d'analyse statistique $\mathrm{R}$ facilite la présentation cartographique de ces disparités.D'autre part, l'absence d'indépendance dans l'allocation des crédits permet de concevoir un modèled'analyse spatiale(relation de voisinage) dans le but non seulement d'étudier les variations dans l'espace mais surtout d'expliquer le caractère inclusif et complémentaire du processus de développement territorial.D'où, les deux axiomes de l'étude :

○ H1 : "La logique de financement des territoires communaux est discriminatoire et renforceles disparités territoriales"

○ H2: "La logique de financement des territoires ne prend pas en compte les interdépendances en termesd'imbrication territoriale et n'aboutit pas à une intelligence territoriale "

Les données qui sont utilisées proviennent des bases de données de la Direction des Finances Locales, du Ministère de l'Intérieur et des Collectivités Territoriales (DFL/MICT) et de l'Institut Haïtien de Statistiques et Informatique (IHSI). Les techniques d'analyse spatiale seront mobilisées afin de procéder à la vérification de nos deux hypothèses.

Le présent article est divisé en trois sections. La première section pose les fondamentaux théoriques de l'approche territoriale du développement en mettant l'accent sur le financement public et les dynamiques locales d'agrégation intermédiaire. Ces deux considérations invitent à des choix politiques raisonnés d'allocation des ressources en vue de développer une intelligence territoriale. La seconde section présente l'analyse statistique spatiale des données de l'étude dans le but de démontrer les disparités socioéconomiques spatiales à travers le phénomène d'hétérogénéité dans le financement budgétaires des territoires. La dernière section discute des interdépendances spatiales et de ses intensités,à travers la relation de voisinage, dans l'analyse de l'allocation budgétaire pour un développement territorialisé en Haïti. Il est question d'expliquer le phénomène d'autocorrélation spatiale qui affecte la logique de financement des territoires et qui invite à développer une nouvelle intelligence territoriale. Enfin, dans la conclusion du travail, les pistes d'amélioration de la stratégie du financement public du territoire seront identifiées pour permettre un meilleur questionnement de la réalité. 


\section{II- Les fondamentaux de l'approche territoriale du développement dans une perspective de mise en place d'une intelligence territoriale}

Dans les différents travaux portant sur l'approche territoriale du développement s'imposent la notion de proximité dans la fourniture de biens et services publicsaux acteur locaux (Pecqueur, 1989 ; Pecqueur et Vicente, 2000). Elle renvoie non seulement aux valeurs morales, aux croyances, aux représentations mais également au mode de coordination ainsi que des modalités de coordination. La proximité vient du partage d'un même point de vue commun par les acteurs localisés. Il est question de représentations d'une situation donnée avec pour effet la coordination entre les acteurs localisés (Providence, 2015) et dont son fondement n'est autre qu'un « champ mutuel d'influences » (Le Breton, 2004).

Or, la proximité cherche à expliquerles interactions des acteurs entre eux, mais également entre les acteurs et leur territoire. D'abord, les interactions entre les acteurs et leur territoire suppose la proximité géographique qui respecte une seule logique de distance. Celle-ci traduit à la fois les principes de déconcentration des services de l'administration centrale et les principes de décentralisation qui suppose une implication des acteurs locaux dans la gestion de leur territoire grâce aux administrations locales. Ensuite, les interactions entre les acteurs soulignent la « proximité organisée » qui comprend deux logiques. Une logique de similitude (la communauté des croyances et de savoirs)qui s'accentue sur les capacités des acteurs à se coordonner à partir d'un ensemble de règles partagées et approuvées (Kirat et Lung, 1995) et une logique d'appartenance(un cadre partagé de règles, de routines et de comportements)consistant à démontrer la capacité d'une organisation à faire interagir ses membres (Torre et Rallet, 2005). La notion de proximité s'inscrit donc dans une dynamique d'interaction entre les acteurs etleurs territoires et entre les territoires.

En fait, l'approche territoriale du développement place les acteurs localisés et les spécificités territoriales au centre de la planification locale. Elle part du système de coordination entre les acteurs impliqués dans le développement de leur territoire et renvoie obligatoirement à la dimension politique de ces interactions de proximité. Cette dimension politique suppose une régulation de l'action collective qui passe par le jeu de pouvoirs hiérarchisés en vue de résoudre certains conflits socioéconomiques et territoriaux (Amable et Palombarini, 2005 ; Providence, 2015). Tout compte fait, ce sont-là les véritables enjeux de la coordination organisationnelle et sociale,s'appuyant sur les intérêts communs des acteurs localisés.

En fait, la construction politique de la proximité découle de la façon dont les acteurs inventent des règles de stabilité susceptibles de transformer les initiatives localesen les fédérant dans des dynamiques de réseaux pour la production et de consommation des biens et services. Le territoire devient lui-même un produit compétitif et remplit lerôle d'accueil des flux humains, matériels et financiers. Ainsi, cela suppose la stabilité et la reproduction d'un système de mise en projet des territoires bien régulé (Providence, 2015). Tout compte fait, la notion de proximité (géographique et organisée), du point de vue d'un «système social», peut être souhaitée ou subie (Searle, 2005). La proximité souhaitée donne souvent naissance à un accord collectif sur les projets et leur financement tandis que la proximité subie témoigne du conflit entre les acteurs localisés sur la nature des liens socioéconomiques et des choix de mise en projet de leur territoire. 
Cette politique de mise en projet des territoires doit s'appuyer sur un climat de confiance entre les différents acteurs afin d'aboutir à une intelligence territoriale. Cette dernière est la combinaison de l'intelligence politique fondée sur la proximité souhaitée et de l'intelligence économique qui renvoie au mode de financement des territoires. Cette approche des relations de proximité, avec sa dimension politique, apparaît comme un fait institutionnel. La proximité dépasse donc la notion de "faible distance ", car les acteurs sont à la fois en interaction (proximité géographique) et se coordonnent cognitivement et politiquement (proximité organisationnelle) dans une logique d'action collective (Providence, 2015). Il s'agit du dépassement d'une simple intelligence économique en se basant sur la compréhension des défis liés à la fourniture de biens et services publics de proximitéqui suppose une planification stratégique et territorialisée du processus de développement national.

En réalité, toute stratégie nationale de développement devrait se base sur une intelligence territoriale propre impliquant une construction harmonieuse des « différents échelons territoriaux ». Elle s'appuie, entre autres, sur une rénovation l'état au moyen de politiques industrielles et sectorielles adaptées et un aménagement conscient du territoire. Un pareil changement de stratégie de mise en projet des territoires se trouve, bien sûr, dans la prise de conscience des modifications des règles des jeux géopolitiques et géoéconomiques mondiales. Ainsi, l'action de l'État, dans la nouvelle configuration globale et pour les pays les plus fragilisés, apparaît indispensable pour créer des réseaux d'acteurs localisés capables de faire face aux défis productifs et commerciaux (Providence, 2015).

L'intelligence territoriale s'intéresse à la prospérité territoriale (création d'emploi et cohésion sociale). Celle-ci cherche à encourager les dynamiques locales et gérer de manière stratégique la diversité des différents échelons territoriaux par la création de réseaux (Pautratet Delbecque, 2009). Cela invite à rendre les territoires visibles (surtout par le financement public) tout enorganisant la vigilance économique pour faciliter leur croissance et protéger de l'emploi. L'intelligence territoriale invite à créer la synergie des pouvoirs publics par despartenariats stratégiques : public/privé, public/groupements associatifs territoriaux etpublic/diaspora. C'est une remise en question des modalités traditionnelles d'intervention publique dans un environnement compétitif.

Dans le cas d'Haïti, l'intelligence territoriale veut associer l'État haïtien à ses partenaires locaux (Collectivités territoriales) pour un meilleur financement du processus de développement des échelons territoriaux. Elle souhaite permettre un impact positif s'articulant autour de la supervision de l'Etat et de l'encadrement financier des échelons territoriaux (création et animation de réseaux).Le principal défi pour les payspauvres comme Haïti est de créer cette dynamique de proximité entre les acteurs et leur territoire, de manière à initier les actions collectives. En ce sens, l'Étatdevrait jouer un rôle de catalyseur du processus de développement local en encourageant les liens de proximité et en établissant les principes de base de la compétitivité territoriale. "Il s'agit de rassembler et de faire coopérer sur un territoire desentreprises d'un même secteur, des services publics, des universités et des centres de recherche » (Pautrat et Delbecque, 2009 : 25).

Le développement d'une intelligence territoriale pose la problématique de l'aménagement du territoire, mais surtout celle concernant de formes de financement des biens et services publics. Elle s'intéresse à la construction de réseaux opérationnels dont le mode privilégié de fonctionnement est transversal et horizontal. La compétitivité territoriale fonctionne au moyen de 
réseaux coopératifs qui dépassent la simple logique ou compétence des entreprises (Providence, 2015). Ainsi, la création de pôles de compétitivité relève d'un choix stratégique de politique de proximité qui place l'intelligence territoriale au centre de toute logique de financement des territoires.

En Haïti,les principes de la décentralisation exigentl'intervention de tous les élus locaux dans la planification du développement économique de leurs territoires. Ainsi, chaque échelon territorial pourrait « afficher leur identité et valoriser leur potentiel à partir de projets portés par les acteurs locaux eux-mêmes » (Chauchefoin, $2001: 6$ ). Les autorités locales chercheraient à définir, à côté de l'État central, des instruments de régulation susceptibles d'accompagner les initiatives d'investissement dans le cadre de l'intelligence territoriale. Malheureusement, elles n'arrivent pas encore à créer des liens forts entre tous les acteurs localisés (tant publics que privés) afin de leur permettre d'augmenter l'intensité de leur relation.

L'intelligence territoriale veut également dire développer une forme de " gouvernance territoriale » capable de concilier les logiquesorganisationnelles des acteurs localisés avec les enjeux géographiques de la planification locale. Il faut pouvoir créer des interdépendances de proximité sous l'arbitrage des collectivités territoriales. Ceci étant dit, l'élaboration des politiques publiques territoriales (à l'échelle départementale) nécessite de s'interroger sur les choix organisationnels de financement des territoires. Au-delà de lanécessité pour les décideurs publics de fournir et d'entretenir des biens et services publics, il est primordial de faciliter le partage d'informationspour la prise en compte des initiatives socioéconomiques locales (Providence, 2015). Les décideurs publics locauxpeuvent faciliter l'établissement de cette " gouvernance territoriale » qui aura pour but de " mieux anticiper les évolutions du territoire grâce à une intelligence plus fine des contextes économiques et des stratégies d'entreprises » (Chauchefoin, 2001:9).

En résumé, deux grandes approchescomplémentaires sont en fait envisageables lorsqu'on veut approfondir les relations de proximité dans la planification du développement territorial. Une première approche par la « compétitivité territoriale » et une seconde par la "mise en réseau des acteurs localisés ». De ce fait, il faut concevoir le territoire comme un espace politique et socioéconomique dynamique, dépassant un simple découpage territorial ou institutionnel (Courlet et Pecqueur, 1992).Leterritoire est dans ce cas une construction historiquement constituée, fondé sur des relations entre les acteurs localisés et sur lequel se déclinent les politiques publiques en actions publiques localisées. Le mérite de cette approche territoriale du développement est de considérer la proximité comme un facteur positif de développement en la ramenant à la notion de « territoire » (Pecqueur et Vicente, 2000 ; Providence, 2015).

Cette étude sur le financement des territoires communaux par la Loi de finance locale 2017-2018 s'appuie sur la notion de proximité afin de mettre l'accent sur le caractère à la fois local et global $\mathrm{du}$ processus de développement national. Elle cherche à le démontrer par la logique de répartition spatiale des crédits qui peut révéler l'opposition des forces d'agglomération et de dispersion dans cette logique de financement de l'Etat haïtien. Ainsi, il est question de comprendre deux phénomènes qui apparaissent dans cette logique de financement des territoires communaux en Haïti : l'hétérogénéité et l'autocorrélation spatiales. 


\section{III- Les disparités spatiales dans la logique de financement des territoires haïtiens par les budgets communaux 2017-2018}

Pour analyser les disparités spatiales dans le financement des territoires communaux, nous faisons appel aux outils de l'analyse spatiale des données géoréférencées afin de démontrer le phénomène d'hétérogénéité spatiale. Ce phénomène suppose que chaque territoire est spécifique non seulement par sa configuration physique mais surtout par les liens de proximité développés par les acteurs localisés. Le financement de chaque territoire devrait répondre à une stratégie de mise en projet de celui-ci à partirdes spécificités locales dans une logique de complémentarité ou de voisinage.

Les variables mobilisées, pour expliquer les disparités spatiales, à travers les documents de Budgets des Collectivités communales, sont les suivantes :

- Les recettes propres composées de la CFPB (contribution foncière de la propriété bâtie), de la patente, des autres recettes fiscales et des recettes non fiscales ;

- La dotation du Fonds de Gestion et de Développement des Collectivités Territoriales (FGDCT) ;

- Les recettes extraordinaires ;

○ Les dépenses de fonctionnement;

- Les dépenses d'investissement ;

○ Les variables démographiques (population, superficie, etc.).

Pour le besoin de cette étude nous utilisons des ratios qui seront traités à partir des packages d'analyse spatiale du Logiciel R, ce qui nous permet de cartographier la réalité étudiée. Ainsi, les différents ratios visent justement à démontrer ces disparités territoriales.

La figure 1 présente la répartition spatiale de la population haitienne selon le nombre aproximatif d'habitant vivant dans chaque commune et leur densité (au kilomètre carré). Ainsi, plus de 70\% des communes ont un effectifs de moin de 100000 habitants. C'est la tendance générale dans tous les département du pays à l'exception de l'Ouest et de l'Artibonite ou le nobre d'habitant par commune semble être très éveler. La seconde grande tendance sur carte regroupe les communes dont la population varie entre 100000 et 200000 habitants. Les six communes de l'aire métropolitaine de Port-au-Prince (Pétion-ville, Delmas, Port-au-Prince, Carrefour, Cité Soleil et Croix-des-Bouquets) accueillent au moins 300000 habitants chacune. Les communes Cap-haitien, Port-de-Paix et st-Marc enregistre un effectif compris entre 200000 et 300000 habitants.

Quand on regarde la distribution pour la variable densité de la population on constate qu'il y a une forte concerntration dans l'aire métropolitaine de Port-au-Prince (entre 10000 et 20000 habitants par Km2 par commune)et le Cap-haitien avec une densité d'environ 5000 habitants par kilomètre carré. Les autres communes du pays affichent une densité beaucoup plus modérée de moins de 5000 habitants au $\mathrm{km} 2$. Cette concertration spatiale aurait l'avantage d'accentuer l'urbanisation mais le manque d'infrastructures publiques et l'absence de véritables schémas ou plans d'aménagement du territoire ont permis le développement des bidonvilles et des constructions anarchiques. En fait, on peut se poser la question de la gestion du territoire en mettant l'accent sur la pression exercée par exemple sur les ressources en eau. 
Figure1 : Répartition communale de la population haïtienne/ la densité

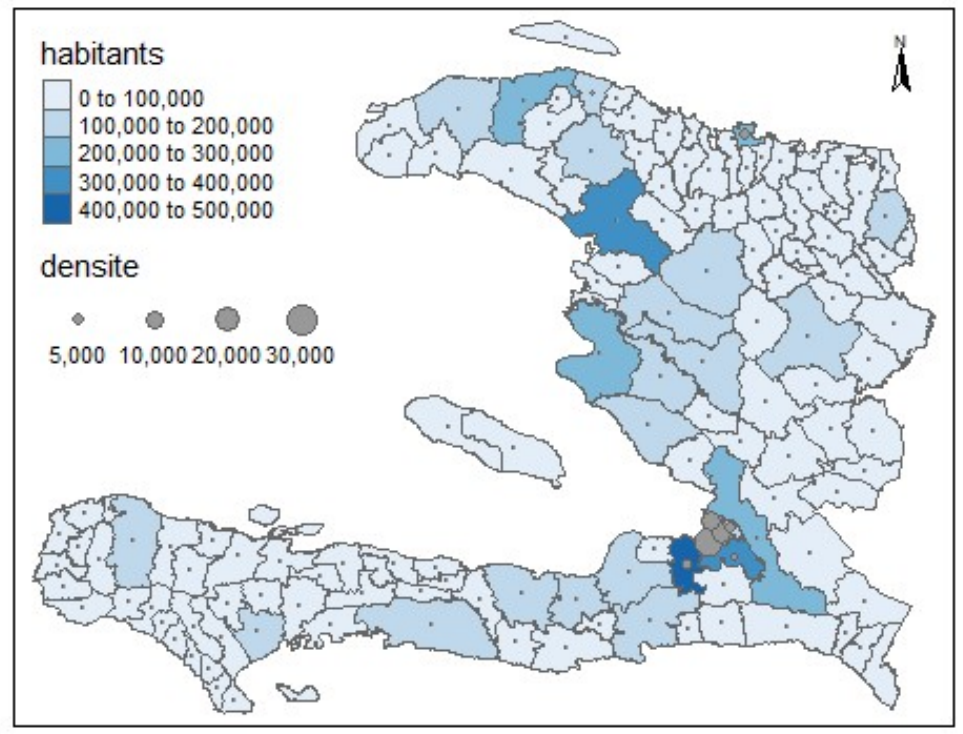

Sources : présentation de l'auteur à partir des données de l'IHSI de 2015

Cette carte (figure 1) permet de mieux comprendre les disparités spatiales en partant de la variable densité qui ramène la population de chaque ville à sa suparficie.Cet indicateur confirme les deux tendances de la répartition communale de la population en ce sens où les communes des deux départements ayant enregistrés la plus forte densité demeurent l'Ouest et l'Artibonite. Ces constats permettent également d'entrevoir les options d'aménagement du territoire haïtien, sachant combien il est nécessaire de lutter contre des phénomènes comme : les constructions anarchiques, la pauvreté et l'exclusion sociale, les crises sociopolitiques récurrentes, etc.

Or la répartition spatiale invite les décideurs publics à mieux penser les actions publiques localisées, découlant des politiques publiques sectorielles, afin de fournir les biens et services publics adaptés aux besoins des contribuables. De ce fait, les actions publiques localisées dérivent en grande partie de la stratégie des collectivités territoriales à exercer les compétences que leur confèrent les Lois de la république. Le financement des collectivités communales doit donc faciliter notre compréhension de la stratégie de l'Etat haïtien à financer le développement territorial. Laquelle stratégie peut également révéler le choix des dirigeants à maintenir, voir même renforcer, les disparités socioéconomiques et territoriales.Avant de présenter les données concernant les dépenses d'investissement et celle sur le fonctionnement des communes, il convient d'analyser la répartition spatiale des recettes propres de ces collectivités communales. Le but étant de comprendre la contribution des acteurs localisés selon le principe de financement des actions publiques par l'impôt.

En effet, la figure 2 discute de la répartition communale en présentant l'interaction entre les recettes propres et le nombre d'habitant pour chaque territoire communal. D'abord les recettes propres sont représentées sur la carte sous l'appellation « financement 0 » dont les modalités sont les suivantes : moins de 5 millions de gourdes, entre 5 millions et moins de 10 millions, entre 10 millions et moins de 20 millions, entre 20 millions et moins de 30 millions, entre 30 millions et moins de 40 millions, entre 40 millions et plus. Ensuite, l'indicateur «nb. Habitants » présentent 
cinq modalités : moins de 200000 habitants, entre 200000 et 400000 habitants, entre 400000 et 600000 habitants, entre 600000 et 800000 habitants et à partir de 800000 habitants. L'analyse croisée de ces deux variables permettra d'approfondir le phénomène des disparités territoriales.

En termes de contribution des citoyens dans le financement de leur territoire les mêmes disparités spatiales sont à déplorer. Les communes du département de l'Artibonite et du département de l'Ouest ont contribué au moins à 10 millions de gourdes de recettes propres. Cependant on doit souligner les communes les plus performantes comme les six communes de l'aire métropolitaine de Port-au-Prince (Carrefour, Port-au-Prince, Pétion-Ville, Delmas, Cité Soleil, Tabarre et Croix-des-Bouquets) et la commune de Cabaret qui ont cotisées à plus de 50 millions de gourdes chacune dans l'Ouest tandis que cette performance a été enregistrée dans seulement deux communes de département de l'Artibonite : Gonaïves et St-Marc. Ce peloton de communes de première catégorie est complété par trois communes chefs-lieux des départements du Nord, du Sud et du Sud-Est, soit respectivement les communes du Cap-Haïtien, des Cayes et de Jacmel.

Figure 2 : Répartition spatiale des recettes propres/la population

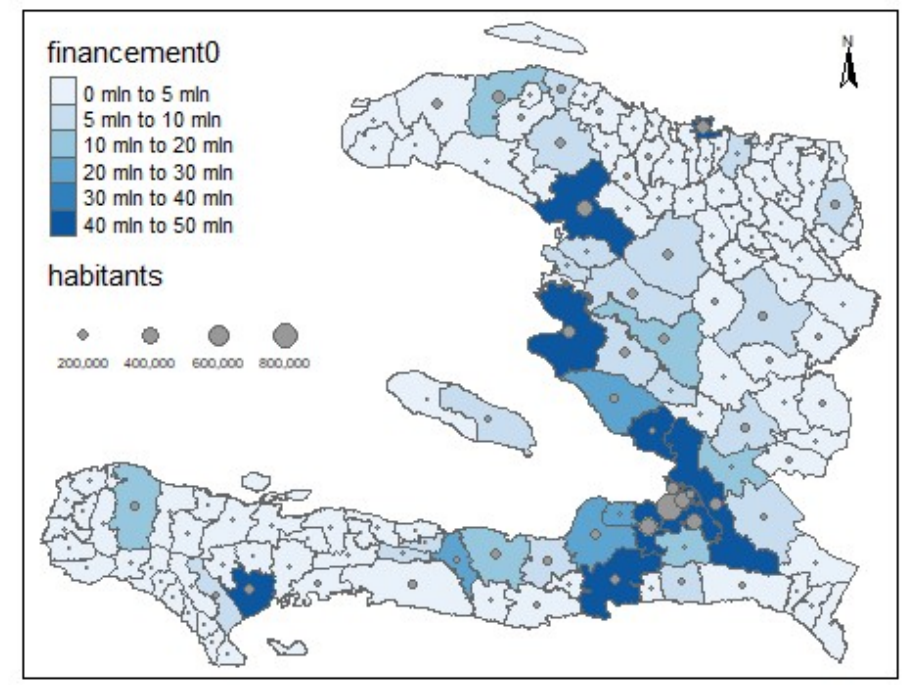

Sources : présentation de l'auteur à partir des données du MICT, budget 2017-2018

Les recettes propres des communes du département de la Grand'Anse n'atteignent pas 5 millions de gourdes chacune à l'exception de Jérémie, le Chef-lieu, qui enregistre environ 20 millions de gourdes. C'est le même constat pour les autres départements (exceptés l'Ouest et l'Artibonite) dont la tendance en termes de recettes propres est de moins de 5 millions de gourdes. Il s'agit encore une fois d'une disparité spatiale grave qui expliquerait la situation de pauvreté extrême dans ces territoires et surtout l'incapacité des décideurs publics à penser le développement de ces localités. Cette disparité invite également à questionner la fourniture de biens et de services publics de proximité aux citoyens de ces territoires. Ce qui suppose de regarder à la fois la répartition spatiale des dépenses d'investissement et celle des dépenses de fonctionnement.

La figure 3 présente la répartition spatiale des dépenses d'investissement par rapport à la population localisée, ce qui donne une idée sur la fourniture de biens publics dans chaque territoire communal. Le nombre d'habitants par commune est représenté par des cercles allant du plus petit, moins de 200 milles habitants, jusqu'à la plus grande agglomération de 800 milles 
habitants et plus.La variable «dépenses d'investissement » est classée suivant six modalités : moins de 10 millions de gourdes, de 10 millions à moins de 20 millions, de 20 millions à moins de 50 millions, de 50 millions à moins de 90 millions, de 90 millions à moins de 120 millions de gourdes et plus de 120 millions de gourdes.

Figure 3 : Répartition spatiale des dépenses d'investissement / la Population

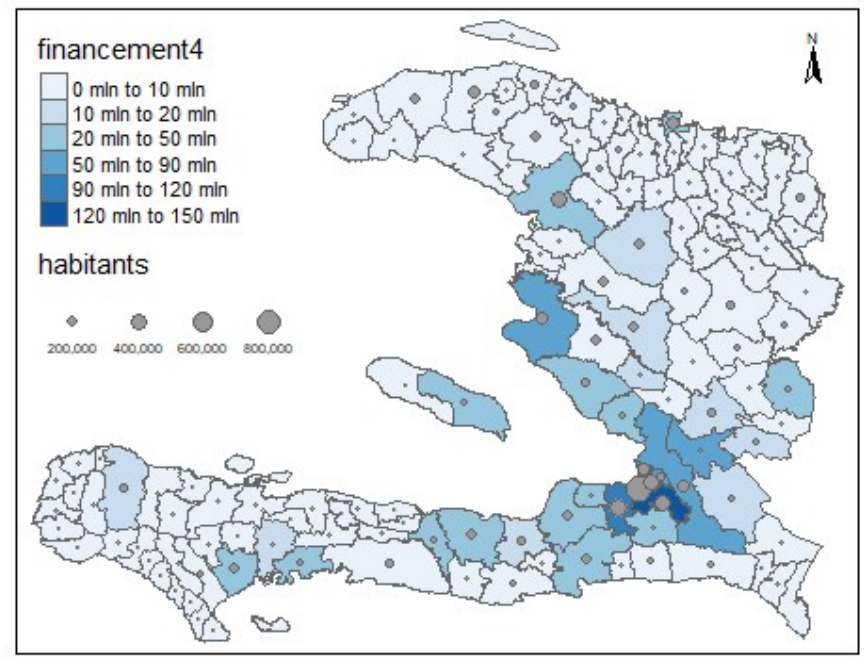

Sources : présentation de l'auteur à partir des données du MICT, budget 2017-2018

Sur la figure 3, on constate une logique de financement de la fourniture des biens publics qui accentue les déséquilibres sur le territoire haïtien. Les communes du département de l'Ouest reçoivent la plus grande part des investissements au moins 10 millions chacune, avec une concentration des investissements dans l'aire métropolitaine de Port-au-Prince. Ainsi, les communes de Pétion-Ville, Delmas, Tabarre, Port-au-Prince et Cité Soleil ont reçu plus de 120 millions de gourdes tandis que Croix-des-Bouquets Gantier et Carrefour ont une dotation comprise entre 90 et moins de 120 millions de gourdes de dépenses d'investissement.

La dynamique de financement des autres départements du pays semble adoptée la règle des disparités spatiales car on constate que même dans certains Chefs-lieux les dépenses d'investissement n'atteignent pas 10 millions de gourdes. C'est le cas des départements du NordEst, du Nord-Ouest et du Centre dans lesquels toutes les communes ont une dotation de moins de 10 millions de gourdes. Cette réalité est légèrement différente dans les départements département du Sud-Est, du Sud, de la Grand'Anse, de l'Artibonite, des Nippes et du Nord oùl'exception se retrouve surtout dans la dotation des Chefs-Lieux qui est comprise entre 20 millions et 50 millions de gourdes, en termes de dépenses d'investissement. Encore une fois les disparités territoriales sont très visibles dans la logique de financement de la fourniture de biens publics qui ne tient pas compte de dynamique de répartition des populations locales sur le territoire national.

La figure 4 présente la répartition spatiale de la dotation communale en termes de dépenses de fonctionnement par rapport au nombre d'habitants sur chaque territoire. Cette variable entend nous renseigner, cette fois, sur la fourniture de services publics de proximité aux acteurs localisés. Les modalités pour la variable sont : moins de 15 millions de gourdes, de 15 millions à moins de 30 millions, de 30 millions à moins de 45 millions, de 45 millions à moins de 60 millions, de 60 millions à moins de 75 millions et de 75 millions à 100 millions de gourdes. La 
figure 4 suppose également qu'on analyse cette répartition spatiale des dépenses de fonctionnement par rapport au nombre d'habitants pour commune afin de constater les disparités spatiales.

Figure 4 : répartition spatiale des dépenses de fonctionnement / Population

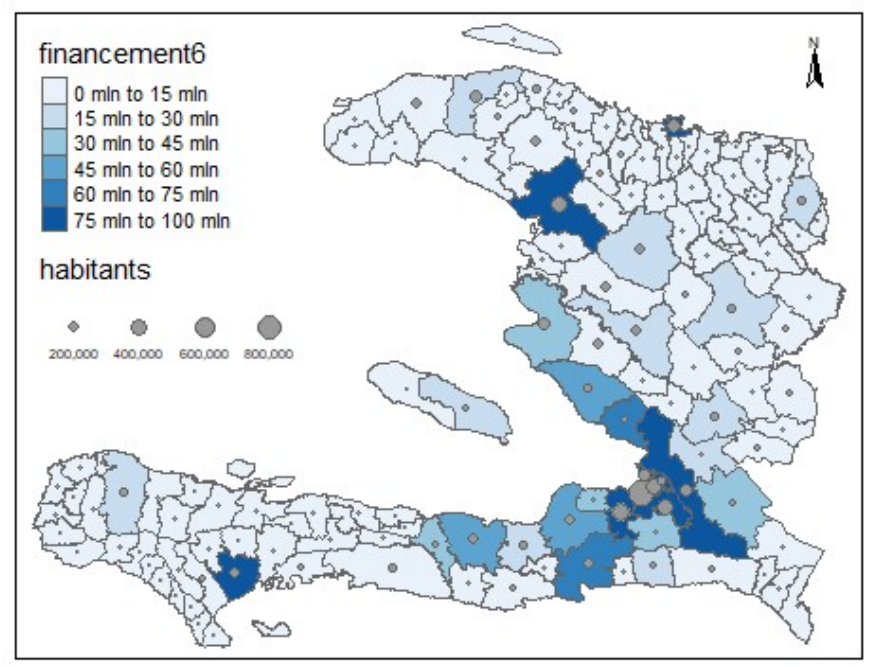

Sources : présentation de l'auteur à partir des données du MICT, budget 2017-2018

Comme dans les cas précédents, la logique de financement des territoires communaux par les crédits de fonctionnement révèle la prédominance de l'aire métropolitaine de Port-au-Prince avec ses six communes qui absorbent chacune au moins 75 millions de gourdes. A l'échelle nationale seules trois autres communes (Cap-Haitien, Gonaïves et Cayes), respectivement Chefs-lieux des départements du Nord, de l'Artibonite et du Sud, reçoivent une dotation similaire. Dans ces trois départements les autres communes, comme dans les autres départements du pays, seulement moins de 15 millions de gourdes sont disponibles pour financer la fourniture de services publics dans chaque commune. Il y a lieu, encore une fois, de constater l'ampleur des disparités spatiales qui souligne le manque de stratégie efficace d'accompagnement du processus de développement des territoires communaux.

Somme toute, les différents indicateurs présentés jusqu'ici révèle une anomalie, en termes de disparités spatiales, dans la planification du processus de développement territorial par les budgets communaux. Il s'agit du phénomène d'hétérogénéité spatiale qui n'est pas prise en compte dans la logique de dotation des crédits communaux. L'omission de ce phénomène se traduit par non seulement l'inadéquation de la fourniture de biens publiques communaux par rapport aux populations localisées mais également par l'inefficacité des services publics de proximité proposés aux contribuables dans ces territoires.Pour compléter cette analyse, il convient maintenant d'identifier le second phénomène spatial qui suppose l'autocorrélation dans le choix d'allocation des ressources aux communautés locales.

\section{IV- L'imbrication territoriale par rapport à la logique de financement des territoires haïtiens par les budgets communaux 2017-2018}

La logique de financement des territoires communaux par les allocations budgétaires, dans le cadre de la fourniture de biens et services publics de proximité, doit être analyser selon les 
principes de complémentarité territoriale et de compétitivité locale. Il s'agit du caractère intégré du processus de développement territorial qui invite à considérer l'unité géographique du territoire haïtien en fonction des spécificités locales. Ainsi, les territoires communaux sont pris dans des relations de voisinage et ces dernières déterminent les dynamiques locales sachant que les phénomènes sociopolitiques et économiques débordent les limites territoriales. D'où la prise en compte de l'imbrication territoriale afin de comprendre les interactions spatiales qui sont considérées, dans la littérature économique sur le développement territorial, comme le fondement du " phénomène d'autocorrélation » (Providence, 2015). Ainsi, ce qui se passe sur un territoire communal dépend de ce qui se passe sur les autres territoires communaux, mais ça décroit avec la distance.

L'autocorrélation suppose que les observations géoréférencées ne sont pas indépendantes et encore moins neutres. Pour comprendre l'intérêt de considérer cette dimension de voisinage, il faut seulement accepter que l'énoncée suivante : le montant total des crédits disponibles pour financer la fourniture de biens et de services publics de proximité est réparti à l'ensemble des territoires communaux suivant une logique de financement arrêtée par l'Etat haïtien ». De ce fait, plus un territoire communal reçoive une plus grande part de dotation initiale moins les autres territoires auront une part importante. Cela suppose que la logique de répartition des crédits peut révéler la stratégie nationale de financement du processus de développement du pays en démontrant les pôles de développement qui sont définis ou choisis par les décideurs publics. Autrement dit, grâce aux relations de voisinage on peut démontrer les dynamiques régionales de management des territoires communaux, tout en identifiant les valeurs atypiques c'est-à-dire, les anomalies dans ces dynamiques régionales de fourniture de biens et services publics de proximité.

Pour mesurer cette interaction entre nos observations, nous allons utiliser une matrice d'interactions spatiales. Il s'agit d'une matrice carrée comportant $\mathrm{N}$ lignes et $\mathrm{N}$ colonnes que l'on nomme $" \mathrm{M} »$ dont les termes diagonaux sont nuls et les termes non-diagonaux varient en fonction des relations de voisinage entre une commune $« \mathrm{i} »$ et ses voisins $« \mathrm{j} »$.

Soit $\mathrm{p}$ le plus proche voisin calculé à partir de la distance des centroïdes des municipalités. La matrice contigüe prend alors la forme générale suivante :

$\mathrm{Mij}=\mathrm{M}^{*} \mathrm{ij}(\mathrm{p}) / \Sigma \mathrm{bM}^{*} \mathrm{ij}(\mathrm{p})$

Avec $\mathrm{M}^{*} \mathrm{ij}$ tel que : $\mathrm{M}^{*} \mathrm{ij}(\mathrm{p})=0$, si $\mathrm{i}=\mathrm{j}$

$\mathrm{M}^{*} \mathrm{ij}(\mathrm{p})=1$, si dij $\leq \mathrm{di}(\mathrm{p})$

$\mathrm{M}^{*} \mathrm{ij}(\mathrm{p})=0$, si $\operatorname{dij}>\operatorname{di}(\mathrm{p})$

Où chaque terme Mij représente la façon dont la municipalité « i » et la municipalité « $\mathrm{j}$ » sont connectés spatialement. Afin de normaliserl'influence du voisinage sur chaque municipalité, cette matrice est standardisée (la somme des lignes est égale à l'unité). Dans ce travail nous allons utiliser des matrices de 1 à 4 plus proches voisins pour mesurer l'intensité des interactions spatiales et identifier le nombre de voisin le plus significatif.

Soit « $\mathrm{Y} »$, le financementtotal (investissement et fonctionnement) dans les communes, si « $\mathrm{M}$ » est notre matrice de voisinage et «Y » le vecteur des $\mathrm{N}$ observation de cette variable spatialisée alors l'autocorrélation suppose : $(\mathrm{MY}) \mathrm{i}=\Sigma \mathrm{jMijYj}$. Il s'agit de la mesure de l'intensité de l'effet 
global sur la i-ième« financement »communal (le ratio: recettes totales sur le nombre d'habitants) des valeurs prises par la variable Y ailleurs dans les autres communes (Jayet, 2001 ; Providence, 2015). Les tests sont tirés du Logiciel « $\mathrm{R} »$.

A ce niveau, l'Indice de Moran est le plus souvent utilisé pour attester de ce phénomène d'autocorrélation spatiale. Statistiquement, il s'agit du rapport de la covariance sur la variance et les économètres spatiaux s'accordent à dire que l'I de Moran est très robuste donc la plus utilisée (Cliff et Ord, 1981 ; Anselin, 1988b ; Oliveau, 2010). Cet indice de Moran soutient que : "plus le nombre de voisins est élevé, plus l'individu a de poids dans la matrice de pondération » (Oliveau, 2010 : 53). Ce qui pousse les chercheurs à standardiser en ligne la matrice pour diminuer ces effets de poids (Le Gallo, 2000 ; Anselin, 1988a).

Pour interpréter les résultats de l'indice I de Moran, on part de la covariance d'un point et de ses voisins, en ramenant le résultat à la variance de l'ensemble des points (Oliveau, 2010). Le résultat peut varier entre -1 (autocorrélation spatiale négative : les valeurs des variables pour les individus voisins sont opposées par rapport à la moyenne) et +1 (autocorrélation spatiale positive : les voisins se ressemblent). À noter que le résultat n'est pas forcément borné entre -1 et +1 . Toutefois, quand il prend la valeur zéro il y a absence d'autocorrélation spatiale négative ou positive. La valeur de l'indice de Moran peut être interprétée comme la part de variance explicable par le voisinage (Providence, 2015). « Un indice de Moran de 0,25 attribuerait ainsi $25 \%$ de la variance aux valeurs dans le voisinage » (Oliveau, 2010 : 54). Pour s'assurer que l'indice de Moran obtenu est significatif, il faut considérer la probabilité (p-value). Si la valeur de probabilité est très faible, pour un intervalle de confiance d'au moins 95\%, elle suppose que la relation de voisinage est très significative. Si elle est très forte cela suppose que la relation n'est pas significative.

Toutefois, la statistique « I » de Moran étant globale, elle ne permet guère d'évaluer la structure, au niveau municipal, de l'autocorrélation. Ainsi, pour comprendre les localisations atypiques ou les poches de non-stationnarité locale : comme une commune riche (en matière de crédits d'investissement et de fonctionnement) entourée de communes pauvres (îlot de richesse), ou à l'inverse, une commune pauvre au milieu des communes riches (mouton noir), etc. Dans cette optique, il convient d'utiliser d'autres indices tels que le diagramme de Moran (Anselin, 1996) ou les indicateurs locaux d'association spatiale (LISA) (Anselin, 1995). Dans ce travail, nous utilisons à la fois le diagramme de Moran et les indicateurs locaux d'associations spatiales dans un souci de continuité et de clarté.

La figure 5 présente les interdépendances dans la logique de financement des territoires pour un (1) plus proche voisin. Trois informations sont données dans cette figure. Une carte modélisant la relation de voisinage pour un plus proche voisin, le test d'autocorrélation spatiale avec les valeurs de l'Indice de Moran exprimant l'intensité de l'autocorrélation et le test de diagramme de Moran soulignant les valeurs atypiques dans les dynamiques régionales.

Les résultats d'Indice de Moran dont la valeur est $\mathrm{I}=0.13$, si on arrondit, confirme qu'il y a effectivement autocorrélation spatiale (positive) entre les observations (Y) dans chaque commune. Ceci dit, la valeur que prend «Y» dans les communes, les financements des territoires communaux pour la fourniture de biens et services publics de proximité, est influence globalement à environ 13\% par le voisinage. Toutefois, la valeur de la probabilité étant élevée, soit $\mathrm{p}$-value $=0.089$, la relation $\mathrm{n}$ 'est donc pas significative. Comme cet indice global d'autocorrélation positive entre les observations ne permet pas de voir les localisations 
atypiques, le diagramme de Moran est proposé afin d'approfondir l'analyse dans cette relation de voisinage.

Figure 5 : Tests de Moran pour 1 plus proche voisin
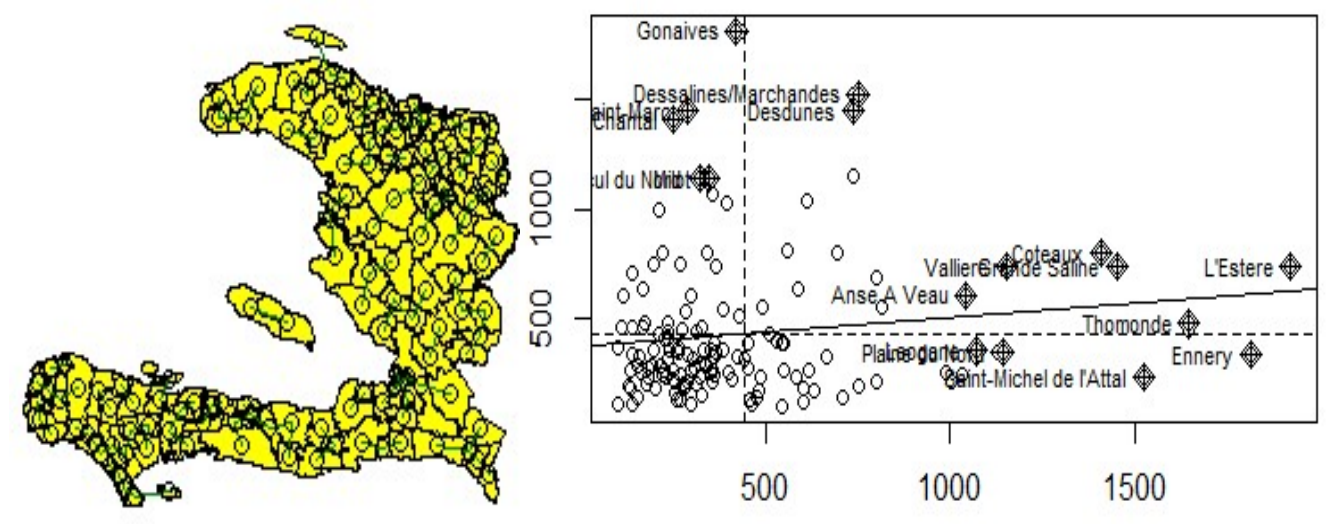

Budget\$Total.Recettes.Population

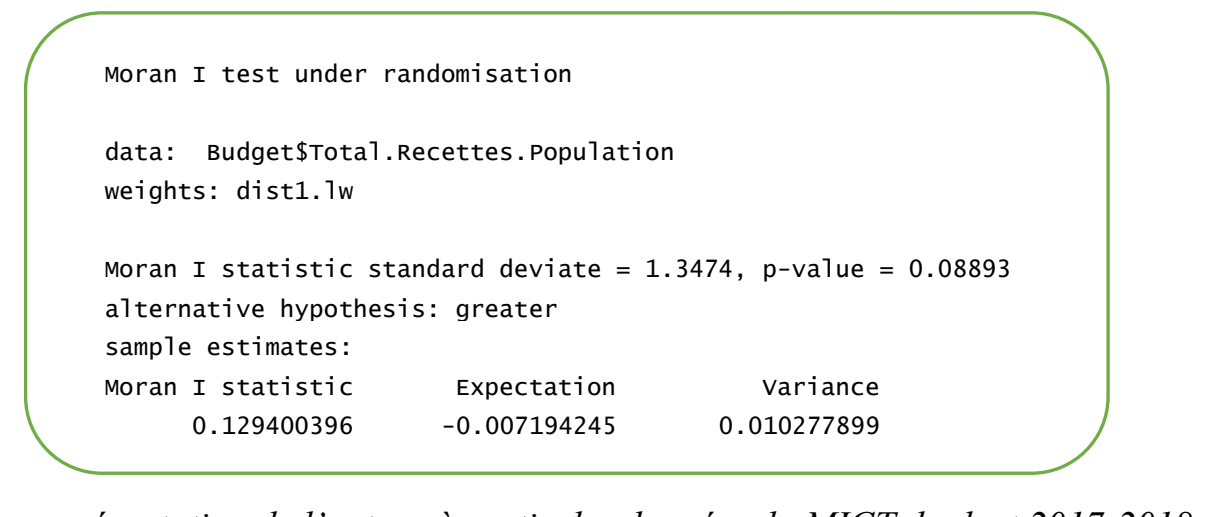

Sources : présentation de l'auteur à partir des données du MICT, budget 2017-2018

En effet, sur la figure 5 on constate la distribution sous la forme de nuage de points des observations de « $\mathrm{Y} »$ dans les différentes localisations. Ainsi, le plan est partagé en quatre (4) cadrant dans un repère orthonormé. La diagonale qui coupe ce repère démontre les deux grandes tendances dans les relations de voisinage: les autocorrélations positives en haut et les autocorrélations négatives en dessous de la diagonale. Dans le diagramme de Moran on peut identifier le premier cadrant noté «HH»(commune associée à une valeur élevée entourée de communes associées à des valeurs élevées) en haut et à droite sur le diagramme, le second cadrant noté « $\mathrm{BB} »$ (commune associée à des valeurs faibles entourées de communes associées à des valeurs faibles) en haut et à gauche, le troisième cadrant noté «HB »(commune associée à des valeurs élevées entourée de communes associées à des valeurs faibles) en bas et à droite et enfin le quatrième cadrant noté « $\mathrm{BH} »($ commune associée à des valeurs faibles entourée de communes associées à des valeurs élevées) en bas et à gauche. 
Sur le diagramme de Moran pour un plus proche voisin le quadrant « $\mathrm{BH} »$ enregistre la plus forte concentration des observations. Cette dernière suppose qu'il y a de profondes disparités spatiales et le financement des territoires communaux n'est pas optimal, ce qui expliquerait le phénomène de paupérisation des localités haïtiennes. Aussi, on peut souligner que les valeurs atypiques se retrouvent dans les trois autres quadrants :

- BB avec des communes comme : Gonaïves, Chantal, Acul du Nord, Saint Marc

- HH avec des communes comme: Marchant Dessalines, Desdunes, Vallière, Coteaux, Anse-à-Veau, L'Estère, Thomonde ;

- HB avec des communes comme : Ennery, Plaine du Nord, Saint Michel de l'Atallaye.

Les communes du quadrant «HB», plus Tomonde représentent une autocorrélation spatiale négative suivant la diagonale. Donc, le diagramme de Moran permet de détecter les localisations atypiques, c'est-à-dire les communes qui dévient du schéma global d'association spatiale. Cela dit, on peut visualiser l'autocorrélation spatiale à partir de la statistique «I » de Moran cependant elle serait plus détaillée quand on va présenter plus loin, les indices locaux de Moran.

La figure 6 suivante reprend la même analyse des tests de Moran pour, cette fois, deux plus proches voisins. La distribution spatiale des observations permet de constater qu'il y a bien autocorrélation spatiale positive entre les territoires communaux, avec un indice de Moran I = 0.193. Autrement dit, l'intensité des interdépendances entre les valeurs de « $\mathrm{Y}$ » (financement des territoires communaux en Haiti) est plus forte que pour la relation de voisinage précédente (un plus proche voisin) et le p-value très faible (0.0042) suppose que la relation est significative. Donc, les valeurs de notre variable sont expliquées à environ $20 \%$ par les valeurs qu'elle prend dans les territoires communaux voisins.

Figure 6: Tests de Moran pour 2 plus proches voisins

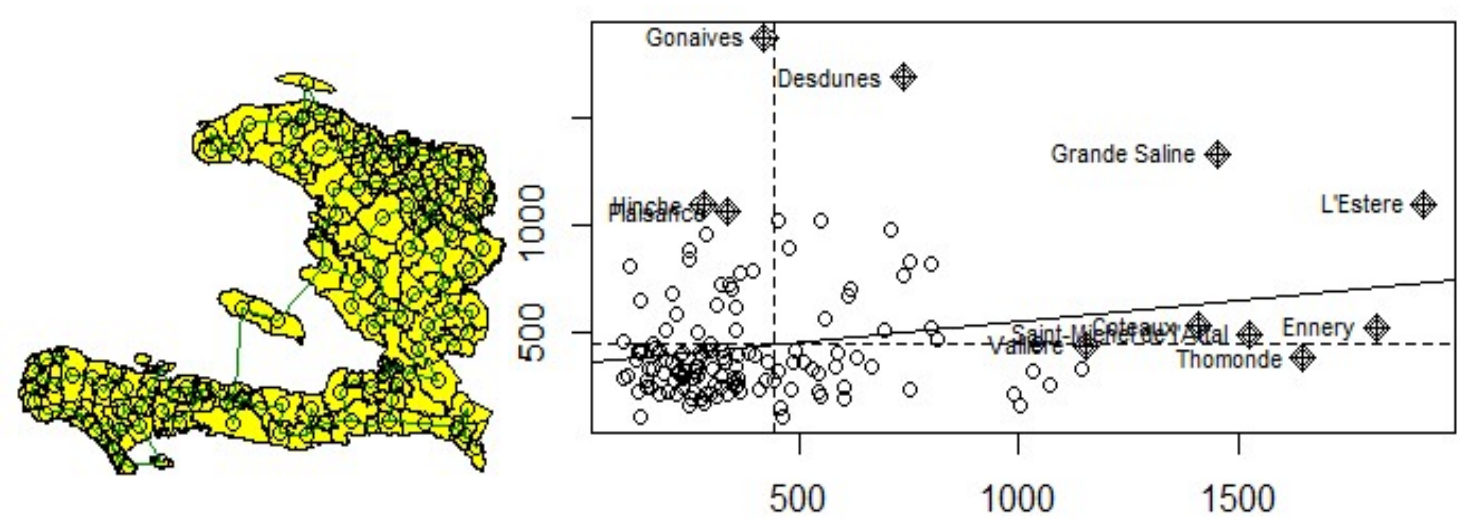

Budget\$Total.Recettes.Population

Moran I test under randomisation

data: Budget\$Tota1.Recettes.Population

weights: dist2.7w

Moran I statistic standard deviate $=2.6394, \mathrm{p}$-value $=0.004153$

alternative hypothesis: greater

sample estimates:

Moran I statistic Expectation Variance

$\begin{array}{lll}0.193451609 & -0.007194245 & 0.005779039\end{array}$ 


\section{Sources : présentation de l'Auteur avec $R$}

Sources : présentation de l'auteur à partir des données du MICT, budget 2017-2018

Or sur le diagramme de Moran, de la figure 6, on remarque la même tendance que précédemment pour le quadrant « $\mathrm{BH} »$ dans lequel il y a une forte concentration. Il s'agit des communes dont les dotations sont élevées qui sont entourées de communes de dotations très faibles. Cependant, on remarque que la diagonale se décale légèrement vers le haut pour expliquer les tendances régionales d'autocorrélation négative. Quant aux valeurs aberrantes ou atypiques, les trois autres quadrants désignent les tendances de territoires communaux :

- BB avec des communes comme : Gonaïves, Hinche, Plaisance

- $\mathrm{HH}$ avec des communes comme: Grande Saline,Desdunes,L'Estère,Saint Michel de l'Atallaye, Coteaux et Ennery ;

O HB avec des communes comme : Vallières et Thomonde.

Les communes du quadrant « HB » (Vallières et Tomonde) et du quadrant BB (Saint Michel de l'Atalaye, Coteaux et Ennery) représentent les valeurs aberrantes d'une autocorrélation spatiale négative suivant la diagonale. Donc, le diagramme de Moran permet de détecter ces localisations atypiques sur le territoire haïtien, c'est-à-dire les communes qui dévient du schéma global d'association spatiale.

Figure 7 : Tests de Moran pour 3 plus proches voisins
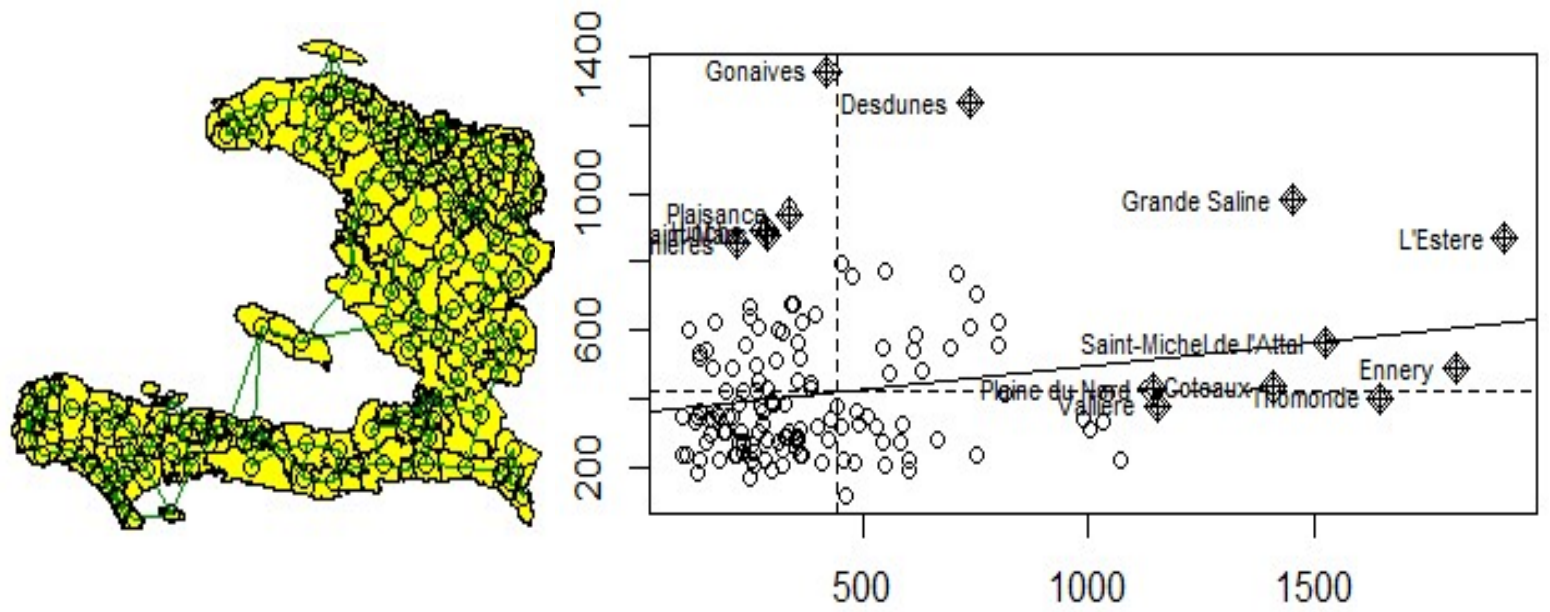

Budget\$Total.Recettes.Population

Moran I test under randomisation

data: Budget\$Tota1.Recettes. Population

weights: dist3.7w

Moran I statistic standard deviate $=2.2787, \mathrm{p}$-value $=0.01134$

alternative hypothesis: greater

sample estimates:

Moran I statistic

Expectation

Variance

0.135422740

$-0.007194245$

0.003917079 


\section{Sources : présentation de l'auteur à partir des données du MICT, budget 2017-2018}

La figure 7 présente les tests de Moran pour les distributions des observations avec une matrice de trois plus proches voisins. La carte donne une idée des connexions spatiales des territoires communaux dans cette relation de voisinage. L'Indice de Moran atteste une nouvelle fois la présence d'autocorrélation spatiale positive entre les observations de notre variables «Y ». Selon la valeur de l'indice de Moran on constate une influence des territoires communaux voisins d'environ $14 \%$. La valeur de la probabilité est de 0.0042 atteste que la relation est significative. Cette intensité est bien supérieure aux données enregistrées pour un plus proche voisin (figure 5), soit environ 13\% (non-significative), mais elle inférieure à l'intensité de 19\% (significative) pour deux plus proches voisins.

Sur le diagramme de Moran de la figure 7, il convient de souligner les résultats suivants :

○ Le quadrant « $\mathrm{BH}$ » enregistre une forte concentration de communes fortement dotées en ressources et qui sont entourées de communes dont les dotations sont très faibles.

- Le quadrant « $\mathrm{BB} »$ pointe du doigt quatre territoires communaux avec de valeurs atypiques positives (Gonaïves, Plaisance, Vallières et Saint Marc)

- Le quadrant « $\mathrm{HH}$ » souligne les valeurs aberrantes dans cinq territoires communaux. Quatre de ces territoires communaux se retrouvent en haut de la diagonale (Desdunes, Grande Saline, L'Estère et Saint Michel de l'Atalaye) et une valeur aberrante dans ce quadrant se retrouvant sous la diagonale (Ennery).

$\circ$ Le quadrant «HB» souligne quatre autres valeurs aberrantes des communes comme Vallière, Plaine du Nord, Coteaux et Thomonde.

La figure 8 présente notre dernier choix de voisinage (4 plus proches voisins) pour analyser l'autocorrélation spatiale des observations sur les différents territoires communaux. L'indice global de Moran souligne, une fois de plus, la présence d'une autocorrélation spatiale positive avec une interdépendance spatiale $13 \%$ (I de Moran $=0.13$ ) en fonction du voisinage. La valeur de la probabilité est peu élevée ( $p$-value $=0.011$ ), cela suppose que la relation est significative. Cette intensité traduit une tendance à la baisse des interactions spatiales entre les territoires voisins par rapport aux deux intensités précédentes pour respectivement deux plus proches voisins $(20 \%)$ et trois plus proches voisins $(14 \%)$.

Sur cette figure 8 on remarque également que le diagramme de Moran présente une légère différence du côté des valeurs aberrantes dans les quadrants « $\mathrm{HH}$ » et « $\mathrm{HB}$ ». La tendance de concentration des observations dans le quadrant $« \mathrm{BH} »$ traduit les disparités territoriales dans le financement des biens et services publics de proximité sur ces territoires. Quant à la distribution des valeurs atypiques on peut noter :

- Dans le quadrant « $\mathrm{BB} »$ on retrouve les communes comme : Gonaïves, Saint Marc et Hinche ;

○ Dans le quadrant « $\mathrm{HH} »$ il y a les communes de: Marchants Dessalines, Desdunes, L'Estère et Grande Saline ;

- Dans le quadrant «HB» on a des communes comme : Saint Michel de l'Atallaye, Coteaux, Plaine du Nord etc. 
Le diagramme de Moran permet d'analyser l'instabilité spatiale locale à partir de la variable spatialement décalée MZt en fonction des variations Zt (Ertur et Koch, 2005). En effet, les valeurs prises par le financement des territoires communaux varient selon la localisation et même en fonction de la stratégie nationale de développement du pays. Ce qui va nous amener à considérer, dans notre analyse des relations de voisinage, la matrice avec deux plus proches voisins. Cette dernière traduit la plus forte influence du voisinage dans la dotation des ressources aux collectivités communales.

Figure 8: Tests de Moran pour 4 plus proches voisins
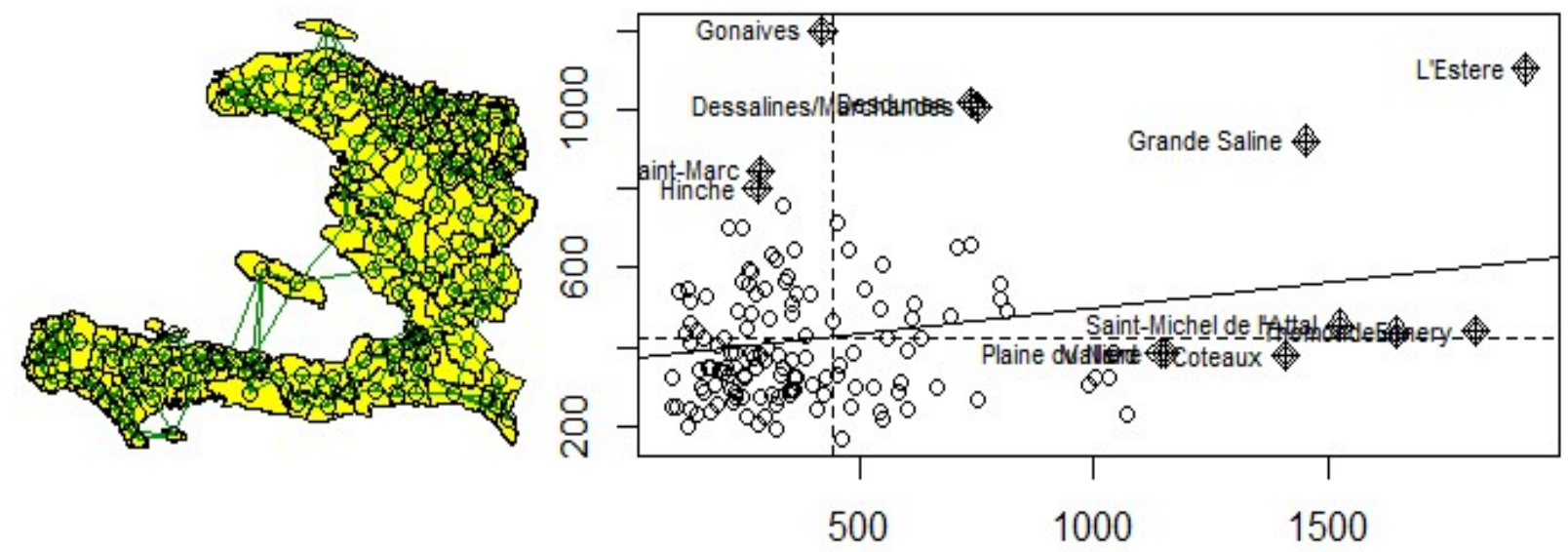

Budget\$Total.Recettes.Population

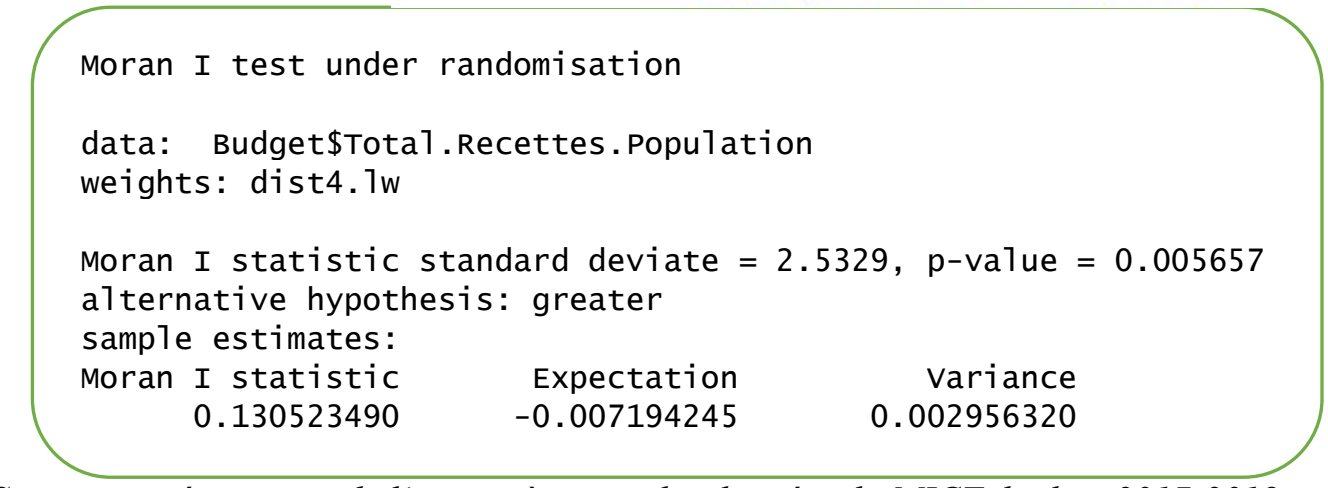

Sources : présentation de l'auteur à partir des données du MICT, budget 2017-2018

Pour compléter cette analyse de l'autocorrélation spatiale positive pour deux plus proches voisins, de la logique de financement des territoires communaux, il faudra faire appel aux indices locaux de Moran. Il s'agit de repérer sur une carte les poches locales d'autocorrélation spatiale qui seront utiles pour expliquer non seulement l'influence de chaque territoire communal mais surtout des groupements de territoire dont les valeurs peuvent être soient très faibles, soient faibles, soient fortes ou encore très fortes et qui tirent le niveau d'autocorrélation spatiale vers le haut.Dans ce cas, les valeurs atypiques des territoires communaux dont les dotations sont différentes de leurs voisins sont devenues visibles parles dynamiques régionales d'association spatiale. Également, il s'agit de constater rapidement les disparités spatiales afin de penser toute action de rééquilibrage des dynamiques territoriales. 
La figure 9 présente la carte de la répartition spatiale des indices locaux de Moran selon l'intensité ou le sens de l'interdépendance entre les territoires communaux voisins. Et comme pour l'indice de Moran global, l'intensité des relations de voisinage varie de]-1 à +1 [ sachant qu'une valeur de l'indice égale à zéro indique une absence d'autocorrélation. Cependant quand l'indice est autour de -1, il y a autocorrélation spatiale négative et elle sera positive quand elle tend vers 1 . En dessous de cette échelle il y aura des valeurs aberrantes négatives tandis qu'audessus se retrouvera des valeurs aberrantes positives.

Sur la carte les valeurs locales aberrantes sont de couleur « rouge » (de -2 à -1), la présence d'autocorrélation locale négative est couleur «orange» (de -1 à zéro), laprésence d'autocorrélation spatiale positive est de couleur «verte» et les valeurs locales aberrantes positives sont de couleur « vert-olive ».

Figure 9 : les indices locaux d'autocorrélation des territoires communaux

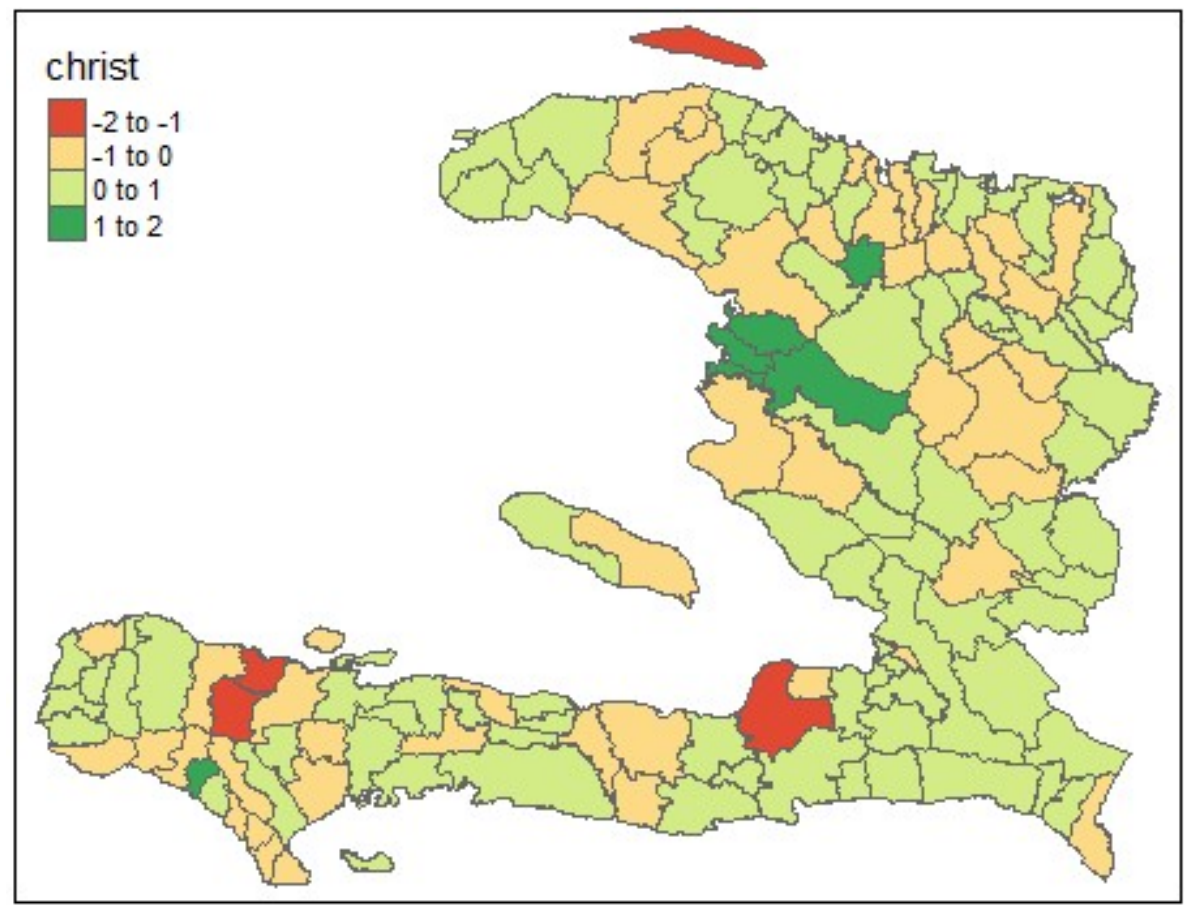

Sources : présentation de l'Auteur avec $R$

Sur la figure 9, les valeurs aberrantes négatives dans trois départements : le territoire de « Ile de la Tortue » dans le Nord-Ouest, le territoire communal de «Léogane » dans l'Ouest, et les communes de «Beaumont» et «Corail» dans la Grand'Anse. Les regroupements de territoires communaux marqués par la présence d'autocorrélation spatiale négative sont distribués dans tous les départements du pays, à l'exception de l'Ouest et du Sud-Est. Les regroupements de territoires communaux marqués par la présence d'autocorrélation spatiale positive sont également distribués sur tout le territoire haïtien. Enfin, les valeurs aberrantes positives se retrouves dans les départements du Sud (la seule commune du « Port-à-Piment ») de l'Artibonite (la Commune de Marmelade et un regroupement des communes de "L'Estère », de « Desdunes », de « Grande Saline » et de « Marchants Dessalines ». 
La lecture de la figure 9 montre bien l'impact du voisinage dans l'appréhension du phénomène de financement des territoires communaux au moyen des crédits budgétaires locaux. Elle nous permet de constater des poches étendues montrant des foyers de faibles financements en « orange » et des zones où les financements sont plus forts en «vert». On voit une autre poche apparaître, qui se trouve dans l'Artibonite en «Vert-olive », où le financement de ces territoires communaux semble optimal.

\section{V- Conclusion}

La prise en compte des phénomènes d'hétérogénéité et d'autocorrélation spatiales a constitué un apport majeur à l'étude des disparités territoriales et du manque d'efficacité de la stratégie de financement des territoires communaux par les crédits budgétaires locaux. Elle a permis de dépasser les conceptions centralisatrices de la stratégie actuelle de développement du pays pour intégrer la dimension spatiale qui place les acteurs localisés et leurs territoires au cœur du processus. Il s'agit d'une remise en question des choix d'allocation budgétaire qui semblent permettre les disparités territoriales et annule toute intelligence territoriale pour un développement intégré des territoires communaux. L'objectif ce travail était d'utiliser les outils d'analyse spatiale pour démontrer le caractère inclusif et complémentaire du processus de développement territorial.

Deux axiomes ont guidé notre analyse spatiale de la logique d'allocation des crédits budgétaires locaux. D'abord, il fallait démontrer le caractère discriminatoire de la logique de financement des territoire communaux par les budgets locaux conduisant à des disparités territoriales. Des ratios sont utilisés et une présentation cartographique de chaque ratio facilite l'étude de ces disparités spatiales. Ensuite, de mesurer l'intensité des interdépendances spatiales des territoires communaux afin de comprendre la portée de l'intelligence territoriale choisie par la logique d'allocation des crédits budgétaires locaux.Pour cela, des tests d'autocorrélation spatiale des observations géographiques sont utilisés pour comprendre l'intensité des interactions spatiales au niveau global (Indice global de Moran) et au niveau local (Digramme de Moran et Indices locaux de Moran).

Concernant le premier axiome, les résultats pour les différentes variables (recettes propres de communes, dépenses d'investissement, dépenses de fonctionnement et population locale) montrent en effet des disparités territoriales profondes dans la logique d'allocation des crédits budgétaires. Quand on ramène les trois premières variables sur le nombre d'habitants(sur les figures 2,3 et 4 ) le caractère discriminatoire de la logique d'allocation apparait. Cette discrimination se manifeste dans la fourniture de biens et services publics de proximitésaux acteurs localisés en donnant la priorité aux territoires communaux de l'aire métropolitaine de Port-au-Prince et à certain Chefs-lieux des départements. La relation prédite dans l'axiome $1 \mathrm{a}$ donc été confirmée par les observations.

Concernant le second axiome, les résultats des différents tests de Moran (les indices globaux et locaux et le diagramme) ont détecté la présence d'autocorrélation spatiale entre les territoires communaux. Du plus proche voisin à quatre plus proches voisins, les intensités des relations de voisinage attestent que les interdépendances territoriales sont très significatives et déterminent des poches de similitude (positive ou négative) capables d'expliquer les dynamiques régionales. Autrement dit, ces résultats démontrent le caractère inclusif et complémentaire de toute logique de financement des territoires communaux à des fins de produire du développement territorial. Quand on ne tient pas compte de ces interdépendances spatiales, la logique d'allocation 
budgétaire ne contribue pas à une véritable intelligence territoriale. Ce qui confirme la relation prédite dans l'axiome 2 de notre travail qui supposait que la logique de financement des territoires communaux par les crédits budgétaires ne tient pas compte des interactions spatiales entre ces territoires.

L'analyse spatiale des dotations communales de crédits budgétaires révèle deux grandes anomalies dans la logique de financement de ces territoires. La première anomalie considère la légitimation des disparités territoriales et socioéconomiques qui vient d'un choix non éclairé des dirigeants de concevoir le territoire haïtien et les relations de proximité entre les acteurs localisés sur une base discriminatoire. D'où la formule : «Tout par et pour l'aire métropolitaine de Portau-Prince ». Il faut simplement prendre comme exemples les constructions anarchiques à travers la République, l'exode rural, etc. La seconde anomalie se retrouve dans le manque vision ou de considération globale dans les stratégies nationales de développement territorial. L'Etat haïtien ne peut pas faire une gestion prévisionnelle et stratégique du territoire car il est trop dépendant des événements conjoncturels.

\section{Bibliographie}

Amable B., Palombarini S., 2004, Une approche néo-réaliste de l'économie politique, Douzième rencontre internationale du GERPISA, comment penser la variété du capitalisme et la diversité des modèles productifs, juin, Paris.

Anselin L., 1995, «Local indicators of spatial association-LISA», Geographical Analysis, vol. 27, no 2, p.93-115.

Anselin L., 1996, «The Moran scatterplot as an ESDA tool to assess local instability in spatial association», In Fischer M., Scholten H.J., Unwin D., Spatial Analytical Perspectives on GIS. Londres: Taylor \& Francis, p.111-125

Anselin L., 1988a, Spatial Econometrics : Methods and Models, Kluwer Academic Publishers, Dordrecht.

Anselin L., 1988b, Lagrange multiplier test diagnostics for spatial dependence and spatial heterogeneity, Geographical Analysis, 20, 1-17.

Anselin L., Bera A., 1998, Spatial dependence in linear regression models with an application to spatial econometrics, dans : Ullah A. et Giles D.E.A. (Eds.), Handbook of Applied Economics Statistics, Springer-Verlag, Berlin.

Anselin L., Griffith D.A., 1988, Do spatial effects really matter in regression analysis?, Papers of the Regional Science Association, 65, 11-34.

Bailey T.C., Gatrell AC., 1995, Interactive Spatial Data Analysis, Longman, Harlow.

Chauchefoin P., 2001/4, l'élu et le manager : quelle gouvernance territoriale dans l'économie mondialisée ? Flux, ${ }^{\circ}$ 46, p. 6-14.

Cliff A.D., Ord J.K., 1981, Spatial Processes : Models and Applications, Pion, Londres. 
Courlet C., Pecqueur B., 1992, "Les systèmes localisés en France : un nouveau modèle de développement », in G. Benko et A. Lipietz (sous la direction de), les régions qui gagent, district et réseaux : les nouveaux paradigmes de la géographie économique, coll. Économiseenliberté, PUF, Paris, p. 81-102.

Cressie N., 1993, Statistics for Spatial Data, John Wiley, New York.

Fotheringham A.S., Brundson C., Charlton M., 2000, Quantitative Geography, Perspectives on Spatial Data Analysis, Sage Publications, Londres.

Griffith D.A., 1988a, Advanced Spatial Statistics, Special Topics in the Exploration of Quantitative Spatial Data Series, Kluwer Academic Publishers, Dordrecht.

Haining R., 1990, Spatial Data Analysis in the Social and Environmental Sciences, Cambridge University Press, Cambridge.

Jayet H., 2001, Econométrie et données spatiales : une introduction à la protique, Cahier d'économie et de sociologie rurales, no. 58-59, p. 105-129.

Kirat T., Lung Y., 1995, "Innovations et proximités : le territoire, lieu de déploiement des processus d'apprentissage ", in N. Lazaric et J. M. Monnier (eds.), Coordination économique et apprentissage des firmes, Économica, Paris.

Le Breton D., 2004, « l'interactionnisme symbolique », Presses universitaires de France, Paris.

Le Gallo J., 2000, Econométrie spatiale 1 : Autocorrélation spatiale, document de travail du LATEC n²000-05, Université de Bourgogne, Dijon.

Le Gallo J., Ertur C., 2000, Exploratory spatial data analysis of the distribution of regional per capita GDP in Europe, 1980-1995, document de travail du LATEC, n²000-09, Université de Bourgogne, Dijon.

Le Loup F. et coll., 2005/4, la gouvernance territoriale comme nouveau mode de coordination territoriale? Géographie, économie, société, Vol. 7, p. 321-332.

Oliveau, S., 2010/1, « Autocorrélation spatiale : leçons du changement d'échelle », L'Espace géographique (Vol. 39), p. 51-64.

Pautrat R. et E. Delbecque, 2009/1, l'intelligence territoriale : la rencontre synergique public/privé au service du développement économique, Revue internationale d'intelligence économique, Vol 1, p. 15-28.

Pecqueur B., 1989, « Le développement local », Paris, Syros.

Pecqueur B. et Vicente J., 2000, « Modèles décentralisés d'interactions et coexistence spatiale des modes de coordination », Revue d'Économie industrielle, no 93, p. 95-116.

Providence C., 2015, « Le système aide-projet mondial et la problématique du développement en Haïti : quelles externalités locales ? », Thèse de doctorat, Université des Antilles.

Searle J., 2005, What is an institution ? Journal of Institutional Economics 1 (1), 1-22.

Torré A., Rallet A., 2005, « Proximity and localization », Regional Studies, n 39 (1), p. 47-60 
Upton G.J.G., Fingleton B., 1985, Spatial Data Analysis by Example. John Wiley, New York. 